\title{
Reflection of hydrogen and deuterium atoms from the beryllium, carbon, tungsten surfaces
}

\section{Meluzova, D. S.}

2019-12-01

Meluzova, D S , Babenko , P Y , Shergin , A P , Nordlund, K \& Zinoviev , A N 2019 , '

Reflection of hydrogen and deuterium atoms from the beryllium, carbon, tungsten surfaces ' ,

Nuclear Instruments \& Methods in Physics Research. Section B: Beam Interactions with

Materials and Atoms, vol. 460 , pp. 4-9 . https://doi.org/10.1016/j.nimb.2019.03.037

http://hdl.handle.net/10138/325130

https://doi.org/10.1016/j.nimb.2019.03.037

cc_by_nc_nd

acceptedVersion

Downloaded from Helda, University of Helsinki institutional repository.

This is an electronic reprint of the original article.

This reprint may differ from the original in pagination and typographic detail.

Please cite the original version. 
Authors preprint of:

Reflection of hydrogen and deuterium atoms from the beryllium, carbon, tungsten surfaces

Meluzova, D. S., Babenko, P. Y., Shergin, A. P., Nordlund, K. \& Zinoviev, A. N., 1 Dec 2019, In : Nuclear Instruments \& Methods in Physics Research. Section B: Beam Interactions with Materials and Atoms. 460, p. 4-9 6 p. 


\title{
Reflection of hydrogen and deuterium atoms
}

\section{from the beryllium, carbon, tungsten surfaces}

\author{
D.S. Meluzova ${ }^{a}$, P.Yu. Babenko ${ }^{a}$, A.P. Shergin ${ }^{a}$, K. Nordlund ${ }^{b}$, A.N. Zinoviev ${ }^{a^{*}}$ \\ ${ }^{a}$ Ioffe Institute, 194021, St.Petersburg, Russia \\ ${ }^{\mathrm{b}}$ Department of Physics, P.O. Box 43, FIN-00014, University of Helsinki, Helsinki, Finland \\ zinoviev@inprof.ioffe.ru
}

Particle reflection coefficients for scattering of hydrogen and deuterium atoms from amorphous beryllium, carbon and tungsten were obtained, which are of interest for thermonuclear reactor physics. For the case of deuterium scattering from tungsten the data were also calculated for polycrystalline and crystalline target. The calculations were carried out by two methods: by modeling the trajectories of the incident particles and by using the binary collision approximation. Interaction potentials between hydrogen and helium atoms and the selected materials were calculated in the scope of the density function theory using program DMol for choosing wave functions. The dependence of the reflection coefficient $R_{N}$ on the potential well depth was found. The results demonstrate a good agreement with the available experimental values.

Keywords: interaction potential, reflection coefficient, tokamak, beryllium, carbon, tungsten

\section{Introduction}

Particle reflection coefficient $R_{N}$ is defined as the ratio of the number of emitted 
particles to the number of particles incident on the solid surface. Further we refer to $R_{N}$ as "reflection coefficient".

The reflection of hydrogen and deuterium atoms from tokamak wall and divertor materials determines the material balance between the plasma and the wall. Reflected atoms collide with plasma ions, and the flux of neutral atoms is produced via the charge exchange. Their detection is used for measuring ion temperature and isotope balance of plasma. Reflection coefficients are also needed to analyze the energy input in the divertor and plasma wall materials.

ITER is planned to work on deuterium-tritium plasma. We chose the scattering of deuterium atom from tungsten as the main object of research. As is known, tungsten was chosen as the divertor material in the ITER tokamak-reactor. Carbon and beryllium are considered as promising materials for the first wall of the reactor. The $100 \mathrm{eV}$ to $10 \mathrm{keV}$ energy range of incident particles was chosen here for studying because these are typical energies of plasma particles. To investigate the isotope effect, we calculated the reflection coefficients of both hydrogen and deuterium atoms scattered from tungsten. In the future, we are also planning to perform calculations for tritium and helium atoms. The latter are products of the thermonuclear reaction.

The experimental data on reflection coefficients for $\mathrm{C}$ and $\mathrm{W}$ is extremely limited $[1,2]$, while that for Be is not available. Computer modeling is widely used to study the scattering of atomic particles from the surface of materials $[3,4]$. The most commonly used code SRIM [5] is based on the binary collision approximation (hereinafter referred to as BCA) which was proposed in [6]. In BCA, scattering of an incident particle from the nearest solid atom is considered, and the particle trajectory is approximated by its asymptotes in the incoming and outgoing parts of the particle trajectory. In our papers [7, 8], the BCA method was used to calculate the reflection coefficients and angular distributions for the scattering of deuterium atoms from various faces of crystalline tungsten using the repulsive potential. The 
presence of a well in the potential causes the attraction of particles at large internuclear distances. This attraction that influences the reflection coefficients has not been considered previously.

The objectives of this work are:

i) checking the influence of the attractive well in the interatomic potential on the calculation of reflection coefficients (for this task, accurate calculations of interaction potentials are needed).

ii) obtaining reflection coefficients for scattering of hydrogen and deuterium atoms from amorphous beryllium, carbon and tungsten, which are of interest for thermonuclear reactor physics.

iii) modifying our code so as to perform the calculation for amorphous, crystalline and polycrystalline targets.

\section{Calculation procedure}

\subsection{Basic methods}

The details of BCA calculations are described in [6-8]. A more accurate method for obtaining reflection coefficients is based on the particle trajectory calculations [9]. This method is free from simplifying approximations used in BCA and makes it possible to take into account simultaneous interactions of the incident particle with the set of target atoms. The calculations using this method are more time-consuming. We calculated reflection coefficients using both BCA and trajectory calculations methods.

The method for calculating particle trajectories from the classical equations of motion was described in [9]. In our trajectory calculations we took into account the atoms located at the distance of $3 \mathrm{~d}$ ( $\mathrm{d}$ is the lattice constant) from the considered particle trajectory point (approximately 80 atoms were taken into account, depending on the crystal face in question). The force was considered to be dependent on the distance between the atoms and was 
calculated as the potential gradient.

For both methods, the starting point of the calculation lies at the distance of $3 \mathrm{~d}$ from the surface. If the particle leaves the solid body, then the calculation stops at the distance of $3 \mathrm{~d}$ as well. Otherwise, the calculation stops when the particle loses $98 \%$ of its energy. With this choice of the start and finish points, the results do not depend on the choice of these parameters. The integration step in the trajectory method was selected from the condition that the error of the particle emission angle is less than $10^{-4} \mathrm{deg}$.

In our calculations, the beam lies in the $(\mathrm{x}, \mathrm{z})$ plane, with the $\mathrm{x}$ and $\mathrm{y}$ axes oriented along the surface and the $\mathrm{z}$ axis being perpendicular to the surface. The glancing angle $\alpha$ is the angle between the incident beam direction and the target plane. In calculating the spectra, the impact area along both $\mathrm{x}$ and $\mathrm{y}$ axes inside the $\pm \mathrm{d}$ interval was scanned with the beam. To verify the calculation accuracy, we sometimes scanned the $\pm 2 \mathrm{~d}$ interval. No differences were noted.

In the crystalline case, spatial positions of atoms are defined by the crystal lattice. We considered scattering from two faces of a tungsten crystal: (100) and (110). Tungsten has a bcc lattice with the constant $d=3.16 \AA$. In the considered case, the $\mathrm{x}$ axis is oriented along the atomic chain. In the case of the (100) orientation, the first layer consists of atoms lying at the vertices of the squares with distances between atoms equal to $\mathrm{d}$ along the $\mathrm{x}$ and $\mathrm{y}$ axes. The second layer is formed by atoms lying in the centers of the cubes. The distance between the layers is $d / 2$. For the (110) orientation, the unit cell consists of five atoms located at the vertices and in the center of the rectangle, the $\mathrm{x}$-axis distances between the atoms remain the same, and the $y$-axis distances, as well as the interlayer distance, increase by a factor of $\sqrt{ } 2$.

In the polycrystalline case, we consider an atomic cluster having a crystal structure whose size exceeds the range of ions in solids. Initial spatial orientation of the crystal plane was chosen randomly.

For crystalline and polycrystalline targets, only the scattering of deuterium atom from 
tungsten was considered.

For amorphous targets, we start with a cluster where the central atom position is randomly distributed in space within the interval of $\pm \mathrm{L} / 2$ along all the three coordinates. Distance $\mathrm{L}$ between two atoms is calculated as $\mathrm{L}=\mathrm{M} /\left(\rho \mathrm{N}_{\mathrm{A}}\right)^{1 / 3}[3]$, where $\mathrm{M}$ is the target atomic mass in amu, $\rho$ is the target density, $\mathrm{N}_{\mathrm{A}}$ is the Avogadro's number. To take into account the existence of the short range order in amorphous body we construct a cluster: the second layer atoms are randomly distributed over a sphere with radius L. All atoms situated above the surface are neglected.

If vectors $\mathbf{x}_{\mathbf{0}}$ and $\mathbf{v}$ represent the spatial position and velocity of the incident particle, vectors $\mathbf{x}_{\mathbf{j}}$ are the coordinates of target atoms, and $\mathbf{b}=\mathbf{x}_{0}-\mathbf{x}_{\mathbf{j}}$, then we can calculate vector $\mathbf{c}=\langle\mathbf{b} \times \mathbf{v}\rangle /|\mathbf{v}|$. Its length is equal to the impact parameter $\mathrm{p}_{0 \mathrm{j}}$. Comparing the values of $\mathrm{p}_{0 \mathrm{j}}$ for all atoms in the cluster, we choose the scattering centre. After that we construct the cluster again choosing the scattering atom to be a centre of the sphere and then rotate this sphere randomly again. Using the same procedure, we find the second scattering centre, and so on. The number of considered trajectories is $10^{6}$.

The displacement of the lattice atoms was taken into account because the velocity of the incident particle is much greater than thermal velocities. Vibrations of the atoms were assumed to be independent, and a shift in the coordinates along three directions was chosen randomly under the assumption of a Gaussian distribution with vibration magnitudes of $0.05 \AA$ for $\mathrm{W}, 0.07 \AA$ for $\mathrm{C}$ and $0.09 \AA$ for Be [3].

As we mentioned above, we performed the calculations using both methods for the DW case for comparison. For the amorphous target, the BCA was used because results provided by both methods differed only slightly.

To take into consideration the influence of the attraction part of the potential on our calculations, in the next section the choice of the interaction potential is discussed. 


\subsection{Interaction potentials}

In [10], interaction potentials for 19 combinations of atoms were calculated in the framework of the density functional theory (DFT) using the DMol software package to obtain a set of molecular wave functions. These data were compared with the potentials obtained from experiments on scattering of particles in gases. It was shown that the applied method gives better agreement with the experiment than the previously used potential models proposed by Moliere [11], Ziegler et al. [12] and Zinoviev[13].

The calculations of the potential for the $\mathrm{H}-\mathrm{W}, \mathrm{C}, \mathrm{Be}$ systems performed by the DFT method showed the presence of a well in the potential, which leads to attraction of particles at large distances (see Fig. 1). The repulsive part of this potential is consistent with the Zinoviev's formula [13]. The repulsive part of the ZBL potential decreases slower with the increase of internuclear distances than our DFT potential and Zinoviev potential. Both the repulsive part of potentials and the presence of well influence scattering cross sections and reflection coefficients.

The depth of the potential well can be related to the molecular bond dissociation energies. The values of the molecular bond dissociation energies for $\mathrm{C}-\mathrm{H}$ and $\mathrm{Be}-\mathrm{H}$ molecules are $\mathrm{U}_{0}=3.50 \pm 0.03 \mathrm{eV}$ and $\mathrm{U}_{0}=2.34 \pm 0.022 \mathrm{eV}$, respectively [14]. In [15], internuclear distances of diatomic molecules are presented. They are $1.1198 \AA$ for $\mathrm{H}-\mathrm{C}$ system and $1.3431 \AA$ for $\mathrm{H}$ Be. The positions of the minima in the dependence of the potential on the internuclear distance, calculated by the DFT method, coincided with these data. The accuracy of $\mathrm{U}_{0}$ calculation using DFT method is estimated to be about $1 \mathrm{eV}$. Therefore, for the attractive part of the potential, we normalized the results obtained by the DFT method to the experimental $\mathrm{U}_{0}$.

We have not found any experimental data on the dissociation energy for the case of $\mathrm{D}-\mathrm{W}$ in the literature. Our DFT results $(4.6 \mathrm{eV})$ are in good agreement with the value $\mathrm{U}_{0}=4.55 \mathrm{eV}$ calculated in [16]. 
All the obtained potentials are adiabatic. Theoretical formulae for interatomic potentials are independent of masses of colliding particles. The influence of isotope mass manifests itself only in the change in the electron reduced mass. This leads to the potential increase by factor $1+\mathrm{m}_{\mathrm{e}} / \mathrm{M}$, where $\mathrm{m}_{\mathrm{e}}$ and $\mathrm{M}$ are the electron and isotope masses, respectively. Therefore we expect relative difference in the potential to be about $0.05 \%$. Let us note that the relative difference between the ionization potential of hydrogen and that of deuterium is less than $0.03 \%$. According to [14], the potential well depths $\mathrm{U}_{0}$ for the C-H and C-D systems practically coincide $(3.50 \mathrm{eV}$ and $3.54 \mathrm{eV}$, respectively). The positions of the minima for these systems are $1.1198 \AA$ and $1.119 \AA$ [16]. For H-Be and D-Be, the minima positions are 1.3431 $\AA$ and $1.3427 \AA$, respectively. This allows the use of the obtained potentials for the H-Be, H$\mathrm{C}, \mathrm{H}-\mathrm{W}$ systems to describe the scattering for the D-C, D-Be, D-W cases. Recommended values of potentials are presented in Table 1.

\subsection{Electronic stopping}

When considering collisions of H, D ions with solids, it is important to take into account electronic stopping. Fig. 2 shows the values of electronic stopping $\mathrm{dE} / \mathrm{dx}$ for the scattering of hydrogen atoms from tungsten, carbon and beryllium. They may be accurately described as a function of the collision energy:

$$
\frac{d E}{d x}=\left(A \cdot \frac{E}{m_{1}}\right)^{n}\left(1+B \cdot \frac{E}{m_{1}}\right)
$$

Values of the electronic stopping given in the NDS database [17] were used for interpolation. This formula can be used at initial energies of 100 to $10000 \mathrm{eV}$ for different hydrogen isotopes. The approximation parameters for the aforementioned systems are given in Table 2 . In formula (1), initial energy $\mathrm{E}$ is expressed in $\mathrm{keV}, \mathrm{dE} / \mathrm{dx}$ is expressed in $\mathrm{eV} / \AA$, and $\mathrm{m}_{1}$ is the projectile mass in amu.

In the case under consideration, both nuclear and electronic stopping are important. The role of electron stopping greatly increases with increasing incident particle energy. 


\section{Results}

\subsection{Influence of the well depth on the reflection coefficients}

Fig. 3 shows the calculated reflection coefficients for the D-W(110) case at the impact energy of $400 \mathrm{eV}$. In [16] it was noted that the potential well depth for molecular complexes $\mathrm{D}-\mathrm{W}_{\mathrm{n}}(\mathrm{n}=1-4)$ ranges from 1 to $5 \mathrm{eV}$, therefore we performed the calculations with the values of well depth $\mathrm{U}_{0}$ varying within the $0-5 \mathrm{eV}$ range. In several cases, we used a model potential with $\mathrm{U}_{0}=0 \mathrm{eV}$ to evaluate the influence of various forms of the repulsive potential. Our results for $\mathrm{U}_{0}=5 \mathrm{eV}$ are close to the values expected for the well depth of $4.6 \mathrm{eV}$ calculated by the DFT method.

As Fig. 3 shows, the attraction of a particle to the surface at large internuclear distances changes the reflection coefficients. If the well depth is $\mathrm{U}_{0}<2 \mathrm{eV}$, at small glancing angles there is a region where the reflection coefficient is close to $100 \%$. This region is absent at $\mathrm{U}_{0}>3 \mathrm{eV}$. It is interesting to note here that $\mathrm{R}_{\mathrm{N}}$ diminishes in the case of deeper wells for all values of the angle, becoming lower than 0.75 for $\mathrm{U}_{0}=5 \mathrm{eV}$ which is close to the value calculated by the DFT method.

In our paper [8] reflection coefficients were calculated using the BCA method and repulsive potential for the D-W(100) case. It was shown (see Fig. 4a) that there is a universal dependence of the reflection coefficient on the perpendicular component of energy $\mathrm{E}_{\perp}=\mathrm{E}_{0} \cdot \sin ^{2} \alpha$. This parameter was proposed by Lindhard [18] to describe the channeling of particles. As can be seen in Fig. 4a, this scaling makes it possible to observe universality of behavior of the curves at small $\mathrm{E}_{\perp}<8 \mathrm{eV}$. This phenomenon is associated with multiple scattering of a particle by chain atoms. As a rule, more than 10 target atoms participate in the scattering, and the scattering angles are to be summed. When the approximation of the atomic chain is inapplicable, the universality of the curve disappears. The peak at $\mathrm{E}_{\perp} \approx 30-40 \mathrm{eV}$ is 
connected with focusing effect on the second layer.

For comparison with the BCA results, we performed trajectory calculations for initial energy of $0.4 \mathrm{keV}$. Fig. $4 \mathrm{a}$ shows that, in the case of the repulsive potential, results obtained using both BCA and trajectory methods are in good agreement.

Our results obtained using the potential with a well depth of $4.6 \mathrm{eV}$ are presented in Fig. 4b. As seen in Fig. 4b, the similarity of the curves for different initial energies is retained but not as strictly as in the case of the exclusively repulsive potential. This can be explained by the fact that the total reflection of the particles does not occur at small glancing angles. The coefficients become lower at small $\mathrm{E}_{\perp}$ because some of the incident particles penetrate deeper into the target due to the presence of an attracting well in the potential. This reduces the probability of a particle leaving the solid. At initial energies of 100 and $200 \mathrm{eV}$, a significant contribution to the reflection coefficient is due to scattering from the first and second layer, as shown in our paper [8]. At energies above $400 \mathrm{eV}$, incident particles penetrate deeper into a solid, and the contribution to the reflection from the first two layers decreases.

For comparison, we have also performed calculations using the BCA method. Qualitatively, the results are in good agreement (see Fig. 4b). Some difference occurs at $\mathrm{E}_{\perp}=20-50 \mathrm{eV}$ when the presence of the well in the potential influences the focusing effect.

Molecular dynamics simulations for the case of low-energy irradiation of $\mathrm{W}$ were performed in [19] for initial energies of $10-1000 \mathrm{eV}$ at normal incidence. These results are consistent with those of our simulation carried out by using the BCA method for the DW(100) case.

\subsection{The comparison with the experiment}

Using the BCA method, we have calculated the reflection coefficients for scattering of deuterium from amorphous tungsten at initial energy $0.4 \mathrm{keV}$ via the repulsive ZBL potential [12] and our DFT potential involving the attractive well. Fig. 5 shows that at small glancing 
angles the difference between the results for different potentials is remarkable. The values obtained with the recommended potential are consistent with experimental results [1]. At small glancing angles, experimental data is absent. To make an additional comparison, we perform the calculations using the standard SRIM code [5]. The results obtained with our code and recommended potential are in good agreement with results given by SRIM.

More extended experimental data exists for the D-C case [2]. Fig. 6 shows that there is a good agreement between the experiment and our results obtained using the recommended potential.

Therefore, we can conclude that reliable data on reflection coefficients for amorphous targets can be obtained by considering the presence of the attractive well in the potential using the BCA method.

\subsection{Comparison of the results for crystalline and polycrystalline tungsten}

As can be seen in Fig. 7, the behavior of reflection coefficients is similar in both cases. The dependences on the collision energy and on the glancing angle are similar. In the case of crystalline tungsten, dips corresponding to particle channeling are observed. The most prominent dips are observed at angles $\alpha=26.6,45,63.4,90$ degrees corresponding to $\operatorname{tg}(\alpha)$ of $0.5,1,2, \infty$. In the case of a polycrystalline target, these dips disappeared because the orientation of the considered cluster of atoms was randomized during simulation.

\subsection{Data for amorphous materials}

Tables 3 show extended data on the reflection coefficients for the H-W, D-W, D-Be and D-C cases. The data for H-W and D-W are very close because the same potential was used. Scattering cross-sections for these cases are very close too. The difference appears because of a difference in their electronic stopping at considered energies. 


\subsection{Energy spectra of scattered atoms}

The characteristic energy spectra of the scattered particles in the D-W case at $\mathrm{E}=1000 \mathrm{eV}$ are shown in Fig. 8. At small glancing angles, the maximum of the distribution is close to the initial energy. As the glancing angle increases, the distribution becomes flatter. The energy distribution of particles is important for calculating the penetration of reflected particles into the plasma and cooling of the boundary plasma layer. In the collisions of reflected atoms with plasma ions, the charge exchange produces fast atoms escaping from plasma. They stimulate wall sputtering, heating and different types of wall erosion.

\section{Conclusions}

Reflection coefficients of hydrogen and deuterium atoms scattering from the amorphous beryllium, carbon and tungsten which are of interest for the thermonuclear reactor physics were calculated. The incident particle energy range of 100 to $10000 \mathrm{eV}$ typical for the ion energies in tokamak plasma was considered. The data is in good agreement with the available experimental results for deuterium scattering from tungsten and carbon.

The calculations were carried out by modeling the trajectories of the incident particles and using the binary collision approximation (BCA) for scattering of deuterium from the $\mathrm{W}(100)$ crystal. Both methods gave similar results, except for the case when there is the focusing effect on the crystal structure.

Potentials of interaction of hydrogen and helium atoms with carbon, beryllium and tungsten targets were calculated by the DFT method using the DMol program for choosing wave functions. For the case of hydrogen, an attractive well in potential was found. The parameters of the potential well were checked using spectroscopy data on the bond dissociation energies and internuclear distances of diatomic molecules. The conclusion has been made that the obtained potential can be used for calculating reflection coefficients for different isotopes. Recommended values of the potential are presented. 
An analytical expression has been suggested to describe electronic stopping of hydrogen isotopes in the considered materials. This formula can be used at initial energies of 100 to $10000 \mathrm{eV}$ for different hydrogen isotopes.

The dependence of reflection coefficient $R_{N}$ on the potential well depth has been revealed. The presence of the attractive potential well strongly influences the values of $R_{N}$.

For the case of deuterium scattering from tungsten, the data was also calculated for polycrystalline and crystalline targets. The dependences on the collision energy and on the glancing angle are similar for both targets. In the case of the crystalline tungsten, dips corresponding to particle channeling are observed. In the case of the polycrystalline target, these dips disappear because the orientation of the considered cluster of atoms was randomized during simulation.

More experimental data, especially for small glancing angles, is needed to verify potential models for ion-surface interaction. 


\section{References}

1. V.V. Bandurko, V.A. Kurnaev et al., J. Nucl. Mater., 176-177 (1990) 630.

2. C.K. Chen, B.M.U. Scherzer and W. Eckstein, Appl. Phys. A, 33 (1984) 265.

3.W. Eckstein, Computer Simulation of Ion-Solid Interactions (Springer, Berlin, 1991), p.104.

4. E.S. Parilis, N.Yu. Turaev, F.F. Umarov, S.L. Nizhnyaya, The theory of medium energy atoms scattering by the solid surface. Tashkent: FAN, 1987. $210 \mathrm{c}$.

5. J.F. Ziegler, J.P. Biersack, SRIM - http://www.srim.org.

6. M.T. Robinson, I.M. Torrens, Phys. Rev. B., 9 (1974) 5008.

7. P.Yu. Babenko, A.M. Deviatkov, D.S. Meluzova, A.P. Shergin, A.N. Zinoviev, Nucl. Instrum. Methods Phys. Res. B., 406 (2017) 538.

8. P.Yu. Babenko, A.N. Zinoviev, D.S. Meluzova, A.P. Shergin, Journal of Surface Investigation: X-ray, Synchrotron and Neutron Techniques, 12 (2018) 520.

9. P.Yu. Babenko, D.S. Meluzova, A.P. Shergin, A.N. Zinoviev, Nucl. Instrum. Methods Phys. Res. B., 406 (2017) 460.

10. A.N. Zinoviev, K. Nordlund, Nucl. Instrum. Methods Phys. Res. B., 406 (2017) 511.

11. G. Moliere, Z. Naturforsch., A2 (1947) 133.

12. J.F. Ziegler, J.P. Biersack, U. Littmark, The Stopping and Range of Ions in Solids, The Stopping and Range of Ions in Matter, Vol. 1. (Pergamon, New York, 1985), 321 p.

13. A.N. Zinoviev, Nucl. Instrum. Methods Phys. Res. B., 269 (2011) 829.

14. B. Darwent, Bond Dissociation Energies in Simple Molecules. NSRDS-NBS. 31. 1970.

15. B.P. Nikolsky, Handbook of Chemist, Vol. 1 (Chemistry Publ. House, Leningrad, 1966), p. 337 (in Russian).

16. L.W. Anders, R.S. Hansen, L.S. Bartell, J. Chem. Phys., 59 (1973) 5277.

17. Nuclear Data Section of the IAEA - https://www-nds.iaea.org/stopping/.

18. J. Lindhard, Mat.- Fys. Medd. Dan. Vid. Selsk. 34 (1965) 1.

19. A. Lasa, C. Björkas, K. Vörtler, K. Nordlund, J. Nucl. Mat., 429 (2012) 284. 


\section{Captions}

Fig. 1. Comparison of the potential calculated by DFT method with the ZBL potential [12] and Zinoviev [13] formula for the D-W case. The DFT method predicts the existence of an attractive well $\mathrm{U}_{0}$ in depth in the potential.

Fig. 2. Electronic stopping for the hydrogen atoms in tungsten, carbon and beryllium targets.

Fig. 3. Reflection coefficients for scattering of $\mathrm{D}$ atoms with the energy of $400 \mathrm{eV}$ from crystalline $\mathrm{W}(110)$ for different depths of the interaction potential well versus glancing angle $\alpha$. The trajectory calculation method was used.

Fig. 4a. Dependence of the reflection coefficients for D-W(100) on the perpendicular energy component for different collision energies for the case of the repulsive potential. The BCA method was used. Data from paper [8] is presented. For comparison, our calculations by the trajectory method at $\mathrm{E}=0.4 \mathrm{keV}$ are given.

Fig. 4b. Dependence of the reflection coefficients for D-W(100) on the perpendicular energy component for different collision energies, obtained by trajectory calculations using the potential with the attractive well depth of $4.6 \mathrm{eV}$. For comparison, calculations by the BCA method at $\mathrm{E}=1 \mathrm{keV}$ are given.

Fig.5. Reflection coefficients for D atoms scattered from amorphous $\mathrm{W}$ calculated by our program code using the ZBL and DFT potentials. For comparison, SRIM calculations and experimental data [1] are shown. 
Fig. 6. Reflection coefficients for $\mathrm{D}$ atoms scattered from amorphous $\mathrm{C}$ calculated by our code using DFT potentials in comparison with experimental data [2].

Fig. 7. Dependence of reflection coefficients $R_{N}$ of $D$ atoms on the glancing angle $\alpha$ for polycrystalline and crystalline $\mathrm{W}(100)$ at various initial energies. The most prominent dips for crystalline target are observed at angles $\alpha=26.6,45,63.4,90$ degrees (marked by arrows) corresponding to $\operatorname{tg}(\alpha)$ of $0.5,1,2, \infty$. In these directions, channeling of bombarding particles takes place.

Fig. 8. Calculated energy spectra of scattered D atoms in irradiating amorphous W with D atoms $1 \mathrm{keV}$ in initial energy at different glancing angles. 


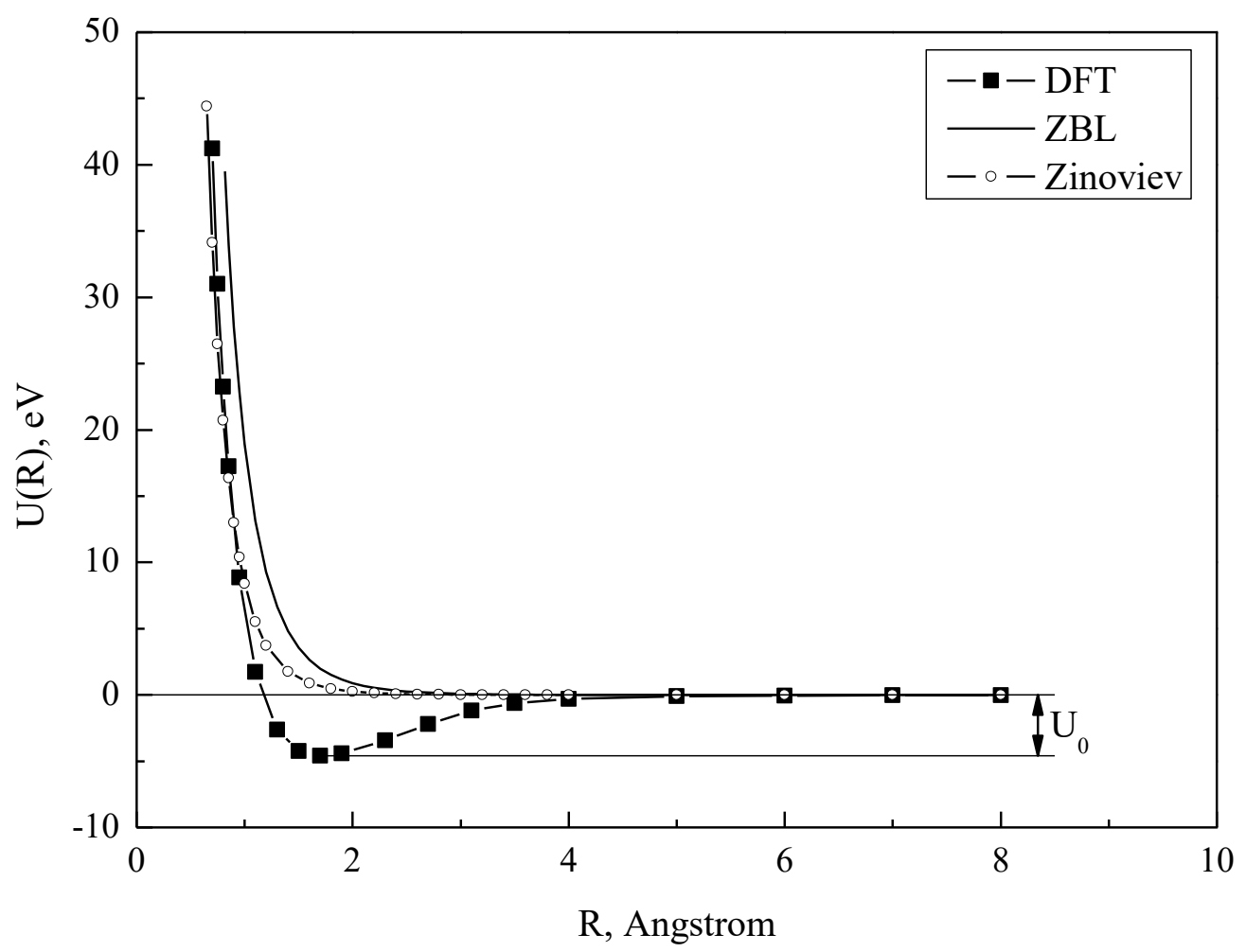

Fig. 1 


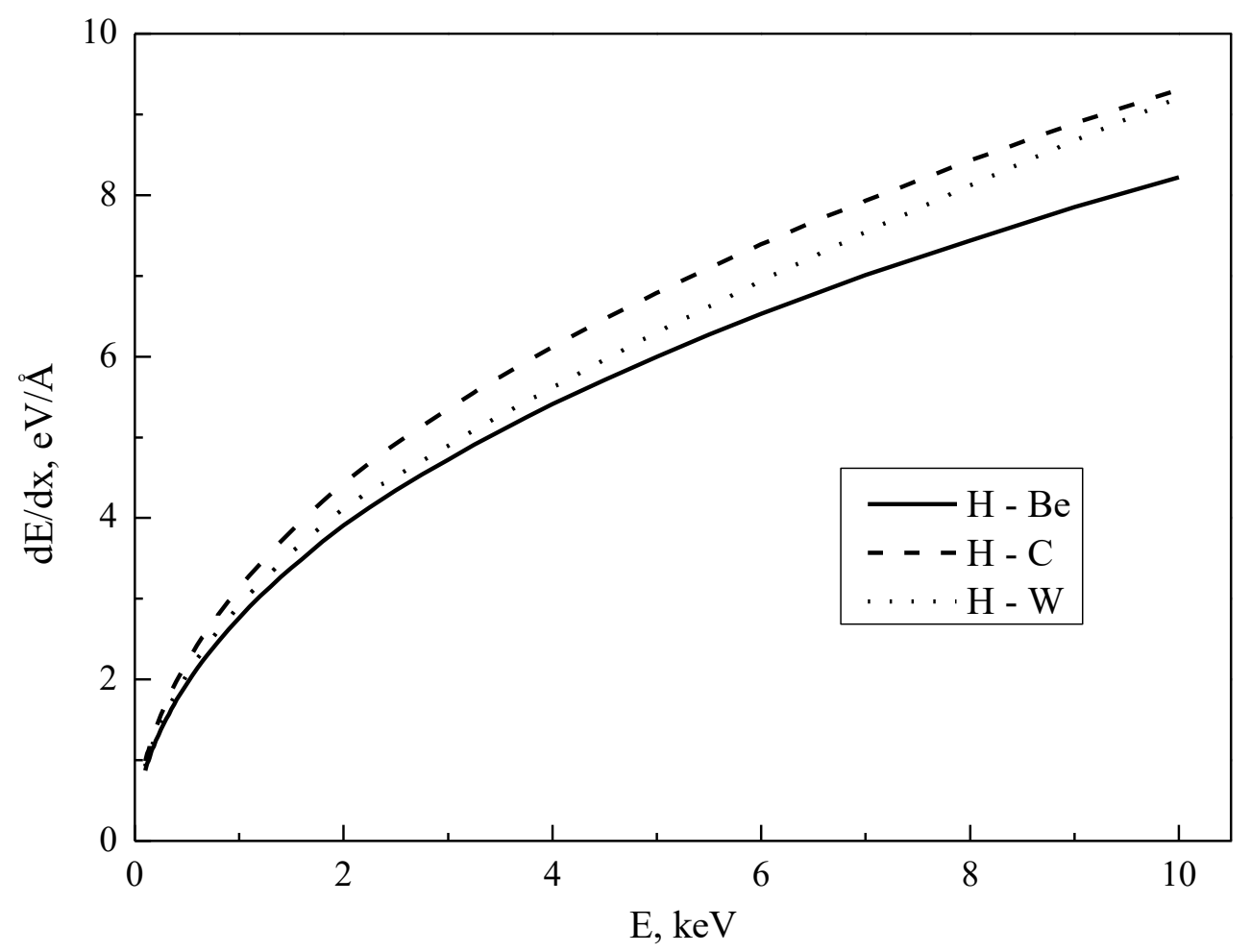

Fig. 2 


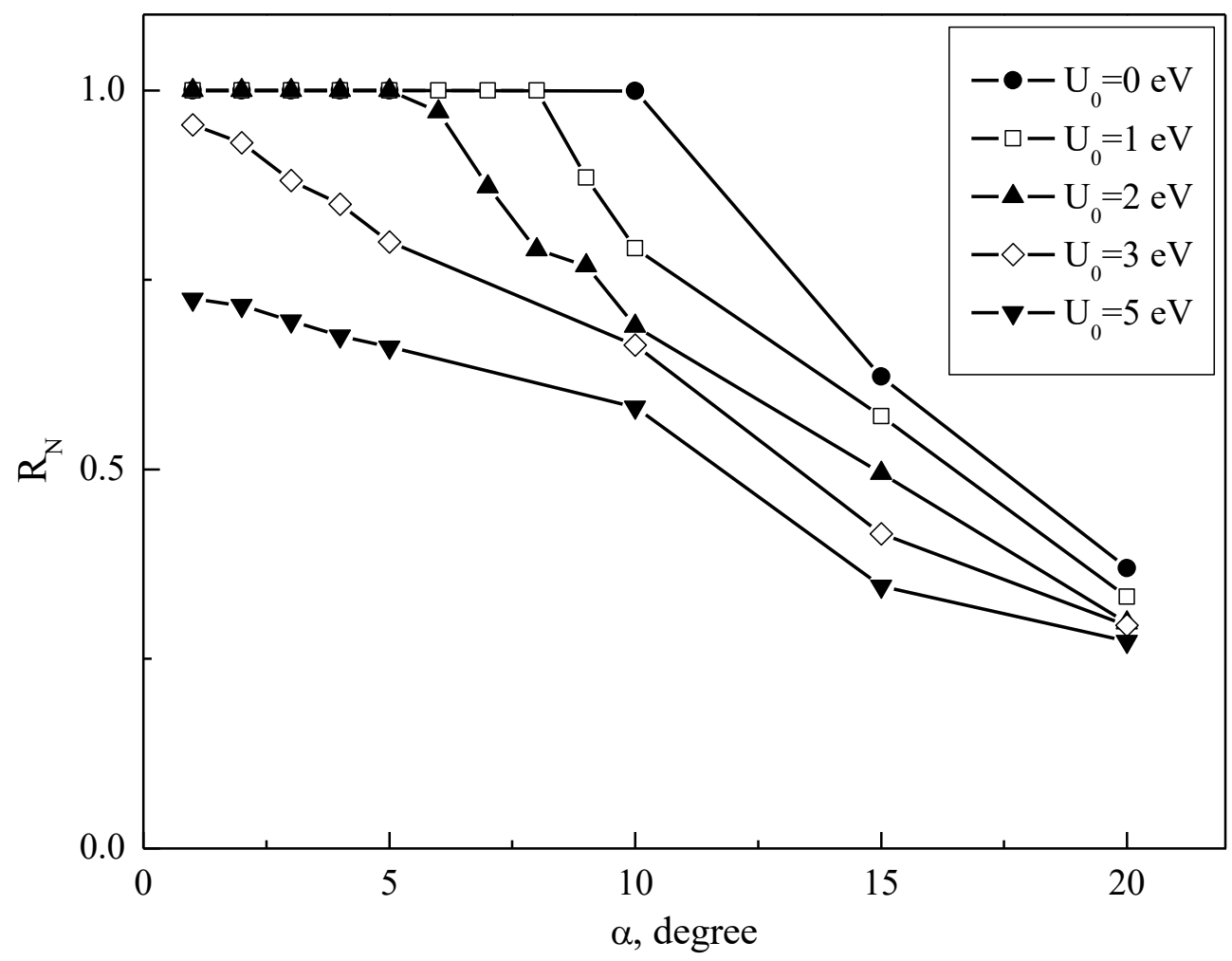

Fig. 3 


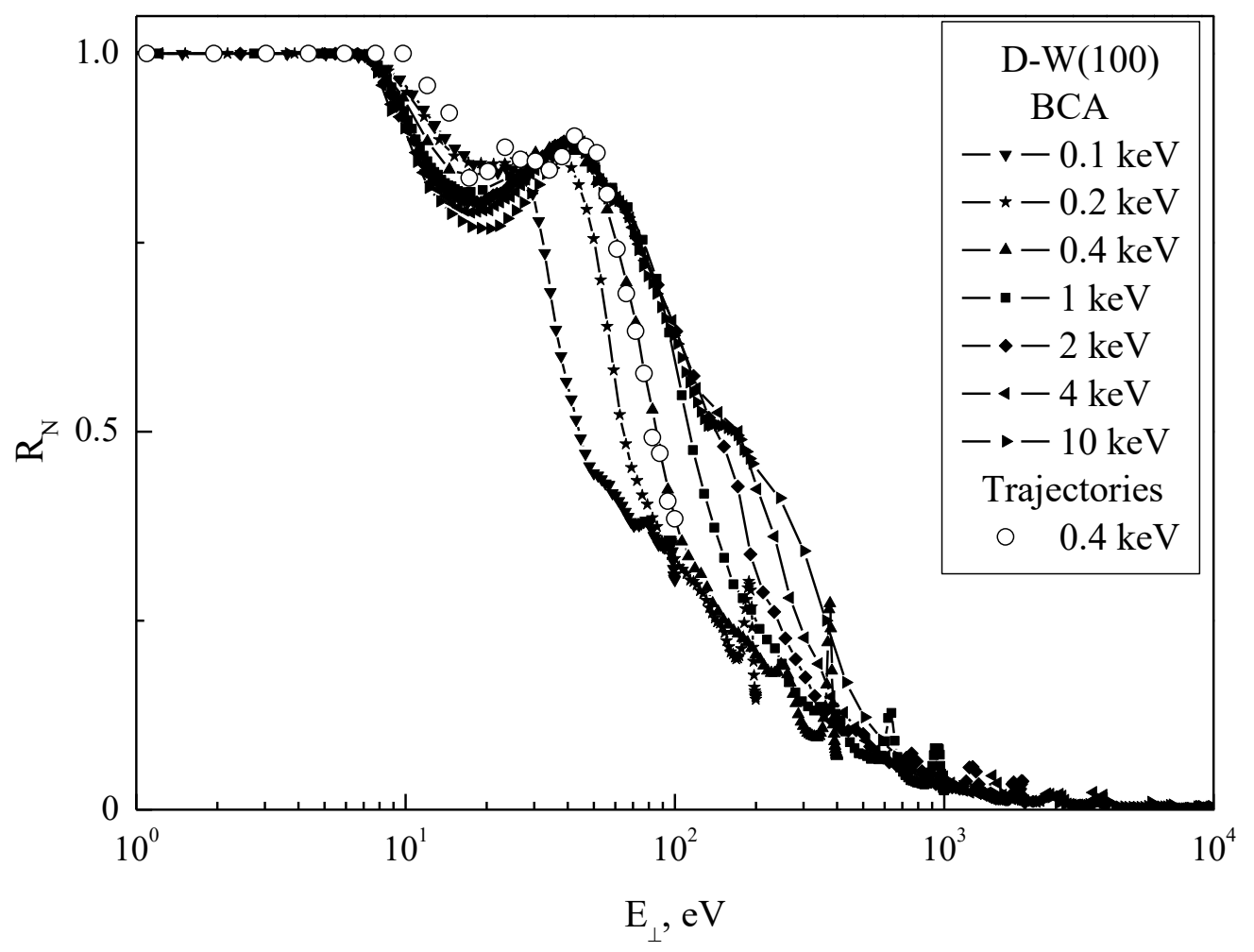

Fig. $4 \mathrm{a}$ 


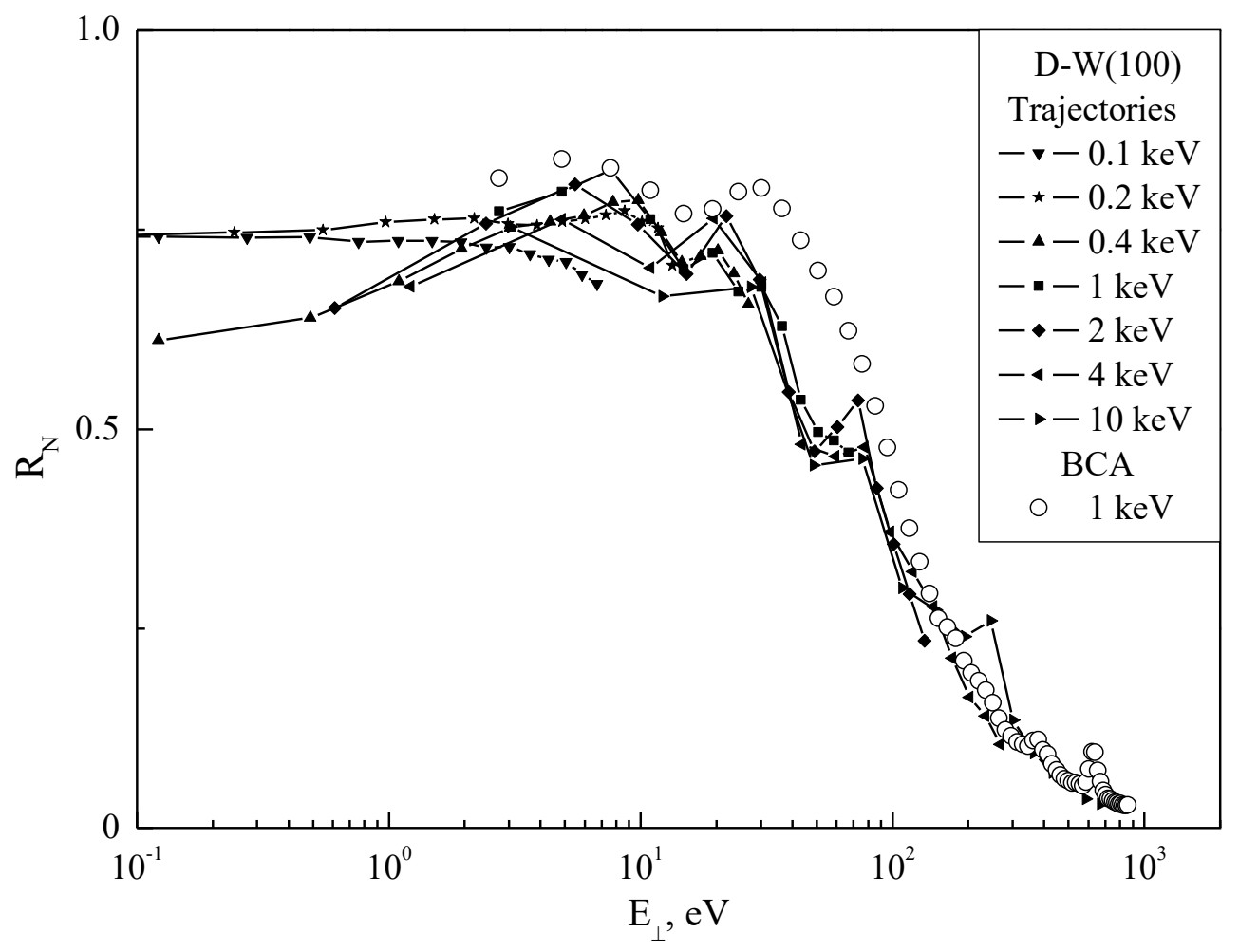

Fig. 4b 


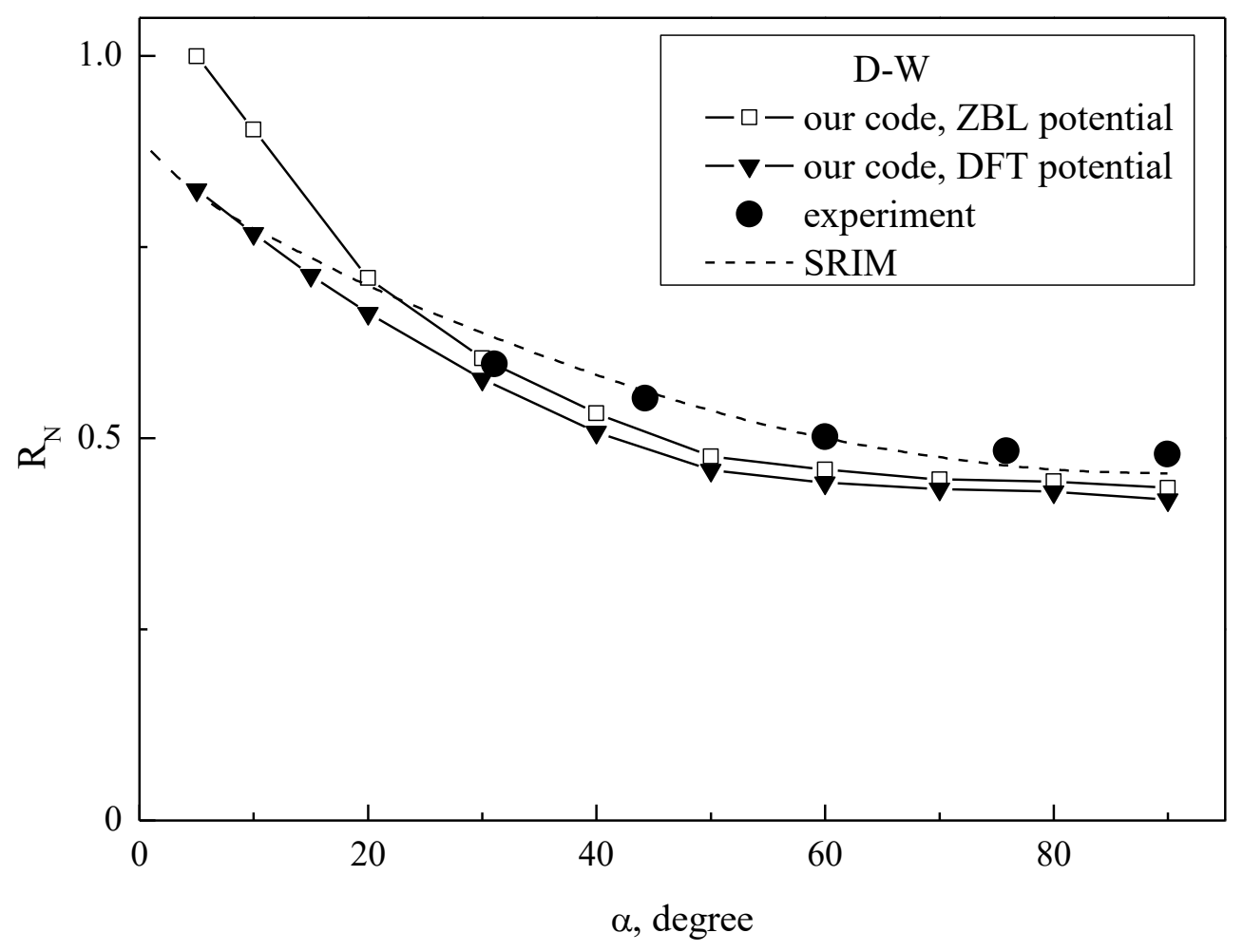

Fig. 5 


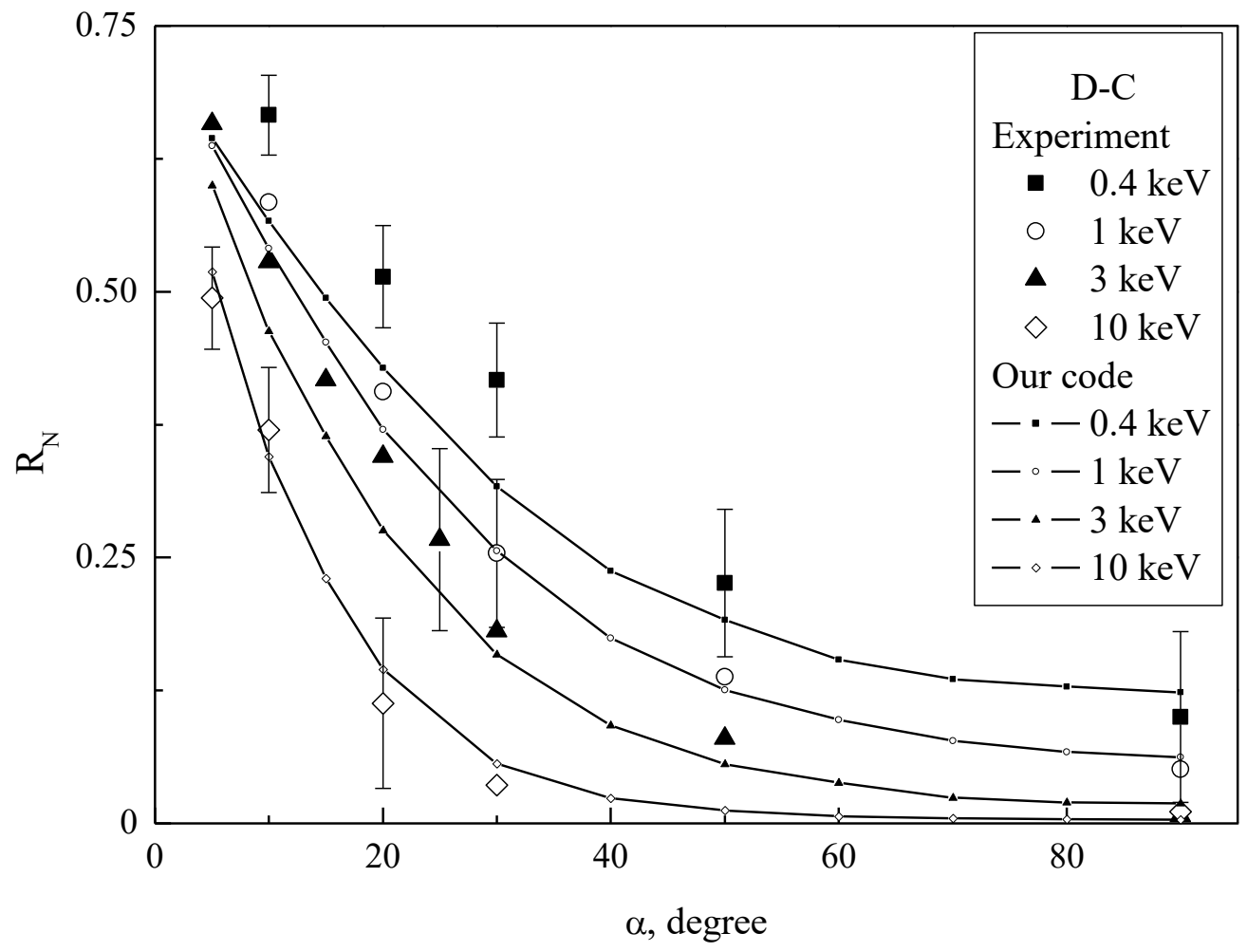

Fig. 6 


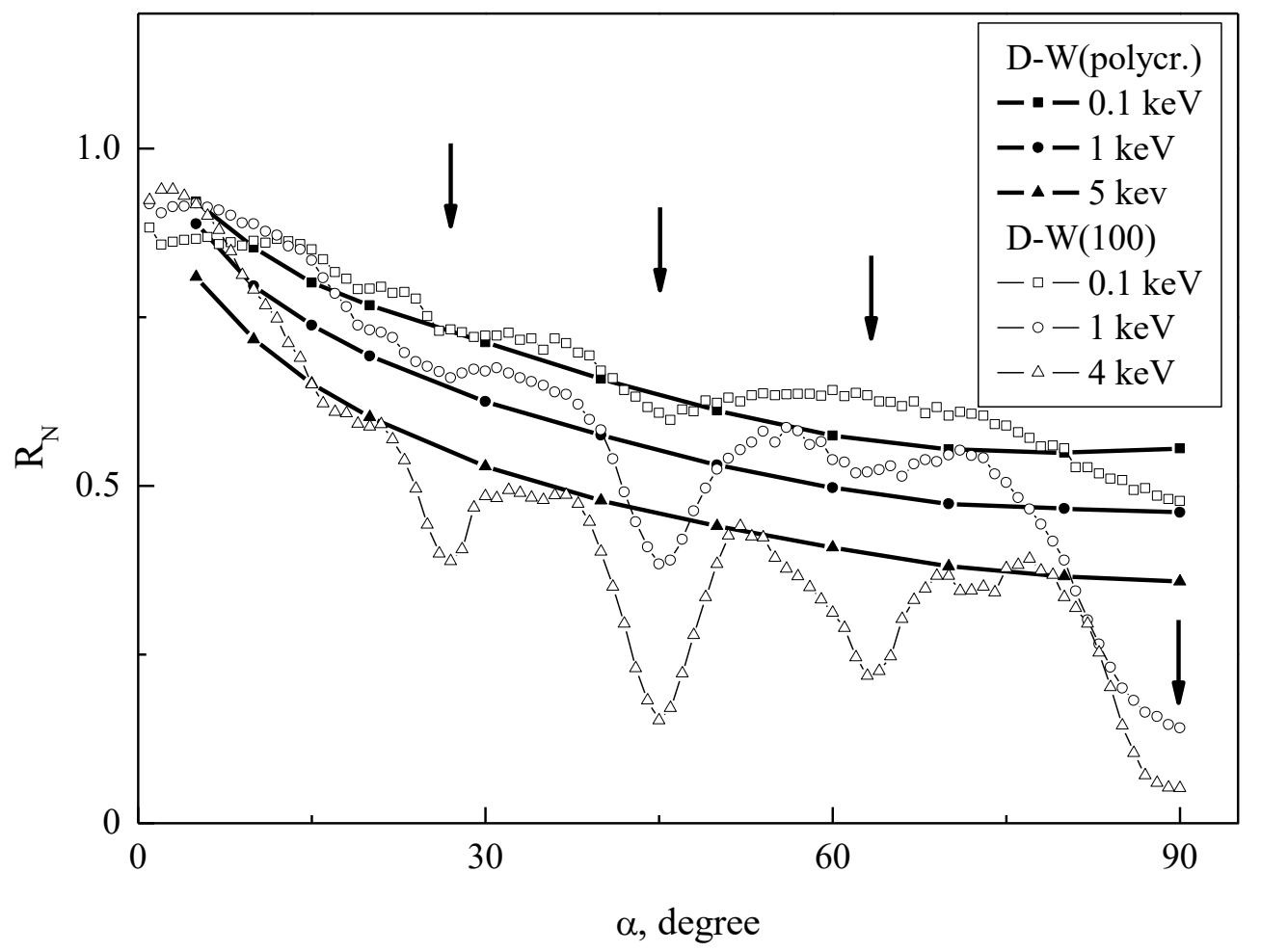

Fig. 7 


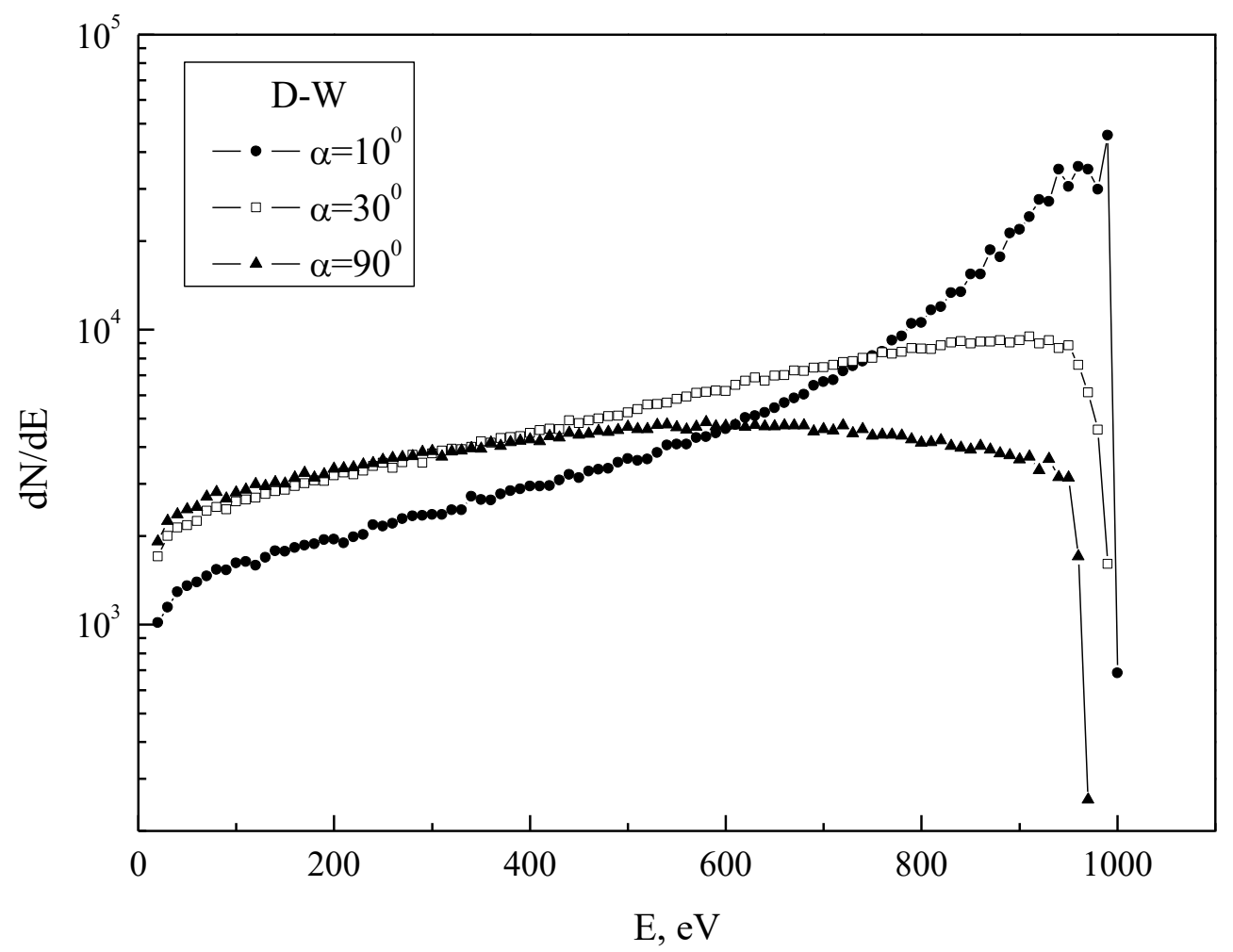

Fig. 8 
Table 1. Recommended interaction potentials for D and He atoms with Be, C, W targets. Values are given in $\mathrm{eV}$.

\begin{tabular}{|c|c|c|c|c|c|c|}
\hline $\mathrm{R}, \AA$ & $\mathrm{D}-\mathrm{Be}$ & D-C & D-W & $\mathrm{He}-\mathrm{Be}$ & $\mathrm{He}-\mathrm{C}$ & $\mathrm{He}-\mathrm{W}$ \\
\hline 0.002 & 28541 & 42760 & 519350 & 57053 & 85464 & 1038000 \\
\hline 0.004 & 14142 & 21161 & 252649 & 28253 & 42269 & 506794 \\
\hline 0.01 & 5504 & 8204 & 94607 & 10974 & 16358 & 189581 \\
\hline 0.02 & 2627 & 3892 & 42855 & 5223 & 7735 & 85897 \\
\hline 0.04 & 1195 & 1755 & 18040 & 2366 & 3470 & 35935 \\
\hline 0.1 & 367.4 & 536.6 & 4676 & 726.9 & 1055 & 9333 \\
\hline 0.2 & 130.4 & 188.4 & 1292 & 260.1 & 365.5 & 2554 \\
\hline 0.4 & 38.01 & 42.62 & 248.1 & 73.41 & 81.27 & 492.7 \\
\hline 0.5 & 21.96 & 19.79 & 131.8 & 42.57 & 41.25 & 259.9 \\
\hline 0.6 & 12.20 & 7.63 & 72.94 & 25.38 & 21.48 & 145.6 \\
\hline 0.7 & 6.108 & 1.012 & 41.22 & 15.64 & 11.43 & 85.54 \\
\hline 0.8 & 2.287 & -1.603 & 23.23 & 10.08 & 6.26 & 51.81 \\
\hline 0.9 & -0.071 & -2.769 & 12.54 & 6.89 & 3.56 & 31.78 \\
\hline 1 & -1.178 & -3.310 & 5.93 & 5.05 & 2.13 & 19.38 \\
\hline 1.1 & -1.826 & -3.500 & 1.743 & 3.93 & 1.329 & 11.54 \\
\hline 1.2 & -2.172 & -3.488 & -0.921 & 3.20 & 0.849 & 6.59 \\
\hline 1.3 & -2.321 & -3.362 & -2.610 & 2.63 & 0.546 & 3.51 \\
\hline 1.4 & -2.340 & -3.175 & -3.637 & 2.12 & 0.349 & 1.653 \\
\hline 1.5 & -2.275 & -2.959 & -4.227 & 1.624 & 0.221 & 0.571 \\
\hline 1.6 & -2.159 & -2.712 & -4.516 & 1.124 & 0.297 & 0.138 \\
\hline 1.7 & -2.012 & -2.442 & -4.599 & 0.833 & 0.288 & -0.013 \\
\hline 1.8 & -1.849 & -2.180 & -4.552 & 0.661 & 0.238 & -0.050 \\
\hline 1.9 & -1.681 & -1.934 & -4.411 & 0.519 & 0.179 & -0.034 \\
\hline 2 & -1.515 & -1.708 & -4.215 & 0.401 & 0.125 & -0.002 \\
\hline 2.2 & -1.204 & -1.316 & -3.744 & 0.230 & 0.048 & 0.048 \\
\hline 2.4 & -0.938 & -1.002 & -3.157 & 0.122 & 0.007 & 0.057 \\
\hline 2.6 & -0.722 & -0.628 & -2.529 & 0.059 & & 0.044 \\
\hline 2.9 & -0.463 & -0.306 & -1.627 & 0.012 & & 0.016 \\
\hline 3 & -0.378 & -0.242 & -1.398 & 0.005 & & 0.009 \\
\hline 3.5 & -0.135 & -0.077 & -0.637 & & & \\
\hline 4 & -0.048 & -0.027 & -0.299 & & & \\
\hline 5 & -0.007 & -0.006 & -0.099 & & & \\
\hline 6 & -0.001 & -0.002 & -0.058 & & & \\
\hline
\end{tabular}


Table. 2 Parameters in formula (2) for describing electronic stopping for various systems. $\mathrm{A}$ is expressed in eV/A $(\mathrm{keV})^{-\mathrm{n}}, \mathrm{E}-\mathrm{in} \mathrm{keV}, \mathrm{B}-$ in $(\mathrm{keV})^{-1}$

\begin{tabular}{|c|c|c|c|}
\hline System & $\mathrm{A}$ & $\mathrm{n}$ & $\mathrm{B}$ \\
\hline $\mathrm{H}-\mathrm{Be}$ & 2.78 & 0.50 & -0.0072 \\
\hline $\mathrm{H}-\mathrm{C}$ & 3.15 & 0.50 & -0.0071 \\
\hline $\mathrm{H}-\mathrm{W}$ & 2.86 & 0.48 & 0.0067 \\
\hline
\end{tabular}


Table 3. Reflection coefficients for various systems. $\alpha$ is a glancing angle, $\mathrm{E}$ is initial energy in $\mathrm{eV}$.

a) $\mathrm{H}$ - amorphous $\mathrm{W}$

\begin{tabular}{|r|r|r|r|r|r|r|r|}
\hline & 100 & 200 & 400 & 1000 & 2000 & 5000 & 10000 \\
\hline 5 & 0.765 & 0.812 & 0.827 & 0.834 & 0.808 & 0.763 & 0.723 \\
\hline 10 & 0.738 & 0.773 & 0.778 & 0.753 & 0.717 & 0.670 & 0.624 \\
\hline 15 & 0.707 & 0.726 & 0.725 & 0.691 & 0.661 & 0.601 & 0.550 \\
\hline 20 & 0.671 & 0.687 & 0.671 & 0.636 & 0.602 & 0.546 & 0.488 \\
\hline 30 & 0.595 & 0.600 & 0.584 & 0.551 & 0.510 & 0.448 & 0.385 \\
\hline 40 & 0.524 & 0.534 & 0.518 & 0.482 & 0.447 & 0.377 & 0.311 \\
\hline 50 & 0.491 & 0.494 & 0.476 & 0.435 & 0.398 & 0.326 & 0.257 \\
\hline 60 & 0.470 & 0.470 & 0.450 & 0.409 & 0.364 & 0.288 & 0.214 \\
\hline 70 & 0.463 & 0.465 & 0.446 & 0.396 & 0.348 & 0.266 & 0.190 \\
\hline 80 & 0.473 & 0.464 & 0.441 & 0.386 & 0.341 & 0.250 & 0.174 \\
\hline 90 & 0.463 & 0.461 & 0.438 & 0.375 & 0.329 & 0.244 & 0.173 \\
\hline
\end{tabular}

b) D - amorphous W

\begin{tabular}{|r|r|r|r|r|r|r|r|}
\hline & 100 & 200 & 400 & 1000 & 2000 & 5000 & 10000 \\
\hline 5 & 0.758 & 0.803 & 0.825 & 0.824 & 0.806 & 0.762 & 0.720 \\
\hline 10 & 0.736 & 0.767 & 0.768 & 0.745 & 0.716 & 0.664 & 0.621 \\
\hline 15 & 0.703 & 0.720 & 0.713 & 0.681 & 0.650 & 0.601 & 0.546 \\
\hline 20 & 0.659 & 0.674 & 0.663 & 0.631 & 0.598 & 0.539 & 0.482 \\
\hline 30 & 0.580 & 0.589 & 0.577 & 0.540 & 0.506 & 0.442 & 0.373 \\
\hline 40 & 0.515 & 0.518 & 0.508 & 0.471 & 0.446 & 0.371 & 0.304 \\
\hline 50 & 0.479 & 0.476 & 0.458 & 0.429 & 0.391 & 0.321 & 0.246 \\
\hline 60 & 0.456 & 0.458 & 0.442 & 0.398 & 0.359 & 0.282 & 0.210 \\
\hline 70 & 0.451 & 0.450 & 0.434 & 0.377 & 0.335 & 0.255 & 0.186 \\
\hline 80 & 0.454 & 0.453 & 0.430 & 0.377 & 0.331 & 0.246 & 0.172 \\
\hline 90 & 0.454 & 0.446 & 0.420 & 0.372 & 0.319 & 0.237 & 0.168 \\
\hline
\end{tabular}

c) D - amorphous C

\begin{tabular}{|r|r|r|r|r|r|r|r|r|}
\hline & 100 & 200 & 400 & 1000 & 2000 & 3000 & 5000 & 10000 \\
\hline 5 & 0.546 & 0.614 & 0.644 & 0.637 & 0.620 & 0.600 & 0.572 & 0.519 \\
\hline 10 & 0.514 & 0.560 & 0.566 & 0.541 & 0.493 & 0.463 & 0.423 & 0.345 \\
\hline 15 & 0.474 & 0.505 & 0.494 & 0.453 & 0.398 & 0.364 & 0.307 & 0.230 \\
\hline 20 & 0.428 & 0.452 & 0.429 & 0.370 & 0.315 & 0.276 & 0.223 & 0.145 \\
\hline 30 & 0.338 & 0.341 & 0.317 & 0.257 & 0.194 & 0.159 & 0.114 & 0.056 \\
\hline 40 & 0.273 & 0.269 & 0.238 & 0.174 & 0.125 & 0.092 & 0.059 & 0.024 \\
\hline 50 & 0.236 & 0.220 & 0.191 & 0.125 & 0.080 & 0.056 & 0.031 & 0.012 \\
\hline 60 & 0.215 & 0.195 & 0.154 & 0.098 & 0.054 & 0.038 & 0.019 & 0.007 \\
\hline 70 & 0.203 & 0.181 & 0.135 & 0.077 & 0.041 & 0.024 & 0.014 & 0.005 \\
\hline 80 & 0.198 & 0.171 & 0.128 & 0.067 & 0.037 & 0.020 & 0.010 & 0.004 \\
\hline 90 & 0.199 & 0.167 & 0.123 & 0.062 & 0.035 & 0.019 & 0.010 & 0.003 \\
\hline
\end{tabular}


d) D - amorphous Be

\begin{tabular}{|r|r|r|r|r|r|r|r|}
\hline & 100 & 200 & 400 & 1000 & 2000 & 5000 & 10000 \\
\hline 5 & 0.622 & 0.667 & 0.686 & 0.653 & 0.616 & 0.544 & 0.483 \\
\hline 10 & 0.576 & 0.588 & 0.566 & 0.511 & 0.459 & 0.374 & 0.292 \\
\hline 15 & 0.503 & 0.495 & 0.470 & 0.403 & 0.351 & 0.258 & 0.172 \\
\hline 20 & 0.435 & 0.416 & 0.381 & 0.317 & 0.260 & 0.170 & 0.098 \\
\hline 30 & 0.316 & 0.290 & 0.250 & 0.196 & 0.141 & 0.071 & 0.031 \\
\hline 40 & 0.225 & 0.202 & 0.171 & 0.124 & 0.079 & 0.032 & 0.012 \\
\hline 50 & 0.179 & 0.155 & 0.124 & 0.078 & 0.048 & 0.016 & 0.006 \\
\hline 60 & 0.154 & 0.127 & 0.095 & 0.055 & 0.030 & 0.009 & 0.004 \\
\hline 70 & 0.141 & 0.114 & 0.077 & 0.042 & 0.022 & 0.006 & 0.003 \\
\hline 80 & 0.135 & 0.105 & 0.071 & 0.035 & 0.017 & 0.005 & 0.003 \\
\hline 90 & 0.134 & 0.102 & 0.067 & 0.034 & 0.016 & 0.004 & 0.003 \\
\hline
\end{tabular}

